\title{
Throughput and Capacity of MIMO WiMAX
}

\author{
Christian Mehlführer*, Sebastian Caban*, José A. García-Naya ${ }^{\ddagger}$, and Markus Rupp* \\ *Institute of Communications and Radio-Frequency Engineering, Vienna University of Technology, Vienna, Austria \\ $\ddagger$ Department of Electronics and Systems, University of A Coruña, A Coruña, Spain \\ Email:\{chmehl,scaban\}@nt.tuwien.ac.at, jagarcia@udc.es, mrupp@nt.tuwien.ac.at
}

\begin{abstract}
In this work, we present physical layer throughput measurement results of a WiMAX link implemented according to IEEE 802.16-2004. The measurements were carried out in an alpine and an urban scenario using the Vienna MIMO Testbed. We compare the measured throughput to three different theoretical bounds: 1) the channel capacity, 2) the mutual information, and 3) a so-called "achievable throughput" which takes inherent system losses into account. Furthermore, the impact of different channel codes and channel estimators on the measured throughput is analyzed.
\end{abstract}

\section{INTRODUCTION}

The potential performance of Worldwide Inter-operability for Microwave Access (WiMAX) has been evaluated by simulations presented in a large number of works, for example [19]. In contrast to [1-8], in [9] Single Input Single Output (SISO) simulation results are presented in terms of physical layer data throughput including link adaptation. In addition to simulations, a few testbed measurements and field trials have been carried out [10-16]. Typically, the results of field trials are throughput values given at a specific receive signal strength. For field trials usually commercial prototypes are used, for which it remains unclear what receiver algorithms are implemented. Also, except [16], previous publications usually lack comparisons between actually measured data throughput and appropriate theoretical bounds.

In [16], we measured the IEEE 802.16-2004 [17, Section 8.3] physical layer throughput and compared it to a so-called "achievable throughput". Back then, we noticed the following shortcomings that motivated new measurements:

- We found that the individual SISO links of the Multiple Input Multiple Output (MIMO) system differ significantly in their average path loss, mainly because of antenna polarization effects. When comparing MIMO to SISO systems, it is therefore necessary to consider all individual SISO links of the MIMO system.

- The measurements in [16] were performed using only the mandatory Reed-Solomon Convolutional Code (RSCC) of IEEE 802.16-2004. It was found that this simple coding scheme causes most (about $5 \mathrm{~dB}$ ) of the Signal to Noise Ratio (SNR) loss compared to the achievable throughput. The optionally standardized Convolutional Turbo Code (CTC) has not been measured.

- In [16], only results from measurements in an urban scenario with a short transmitter-receiver distance were presented.

These points were now addressed in two new measurement campaigns with a very realistic transmitter and receiver placement. The first campaign was carried out in an alpine valley in Austria where the transmitter and the receiver were placed at a distance of $5.7 \mathrm{~km}$. In this alpine set-up, scattering objects existed only in the immediate vicinity of the receiver. Hence, the propagation channel had a very small mean Root Mean Square (RMS) delay spread of about $260 \mathrm{~ns}$. The second campaign was carried out in the inner city of Vienna, Austria, where the transmitter and the receiver were placed at a distance of $430 \mathrm{~m}$. In this urban set-up, the propagation conditions were non-line-of-sight with a large mean RMS delay spread of approximately $1 \mu \mathrm{s}$.

Additionally to the RS-CC and the CTC, also a regular Low Density Parity Check (LDPC) code with variable node degree of $d_{\mathrm{v}}=3$ was measured. At the receiver, three different types of channel estimators were implemented: 1) a geniedriven channel estimator that uses all data symbols for channel estimation, 2) the Approximate Linear Minimum Mean Square Error (ALMMSE) channel estimator of [18], and 3) the Least Squares (LS) channel estimator.

The measured data throughput is compared to three different theoretical bounds: 1) unconstrained channel capacity, 2) mutual information, and 3) achievable throughput. All of them are defined in Section II. Section III briefly explains the measurement setup utilizing the Vienna MIMO Testbed. Measurement results are presented in Section IV and our conclusions in Section V.

\section{Theoretical Performance Bounds}

In this section, we define the theoretical performance bounds for the measured data throughput. These bounds are calculated from the channel coefficients obtained by the geniedriven channel estimator in the measurements. We denote by $k=1 \ldots K$ the subcarrier index of the Orthogonal Frequency Division Multiplexing (OFDM) system, by $r=1 \ldots R$ the channel realization index, and by $a=1 \ldots A$ the transmit power attenuation index.

\section{A. Channel Capacity}

For calculating the channel capacity of a frequency selective MIMO channel $[19,20]$, consider the singular value decomposition [21] of the channel matrix $\mathbf{H}_{k}^{(r, a)}$ scaled by the standard deviation $\sigma_{\mathrm{n}}$ of thermal additive white Gaussian 
noise impairment:

$$
\begin{aligned}
\frac{1}{\sigma_{\mathrm{n}}} \mathbf{H}_{k}^{(r, a)} & =\mathbf{U}_{k}^{(r, a)} \boldsymbol{\Sigma}_{k}^{(r, a)} \mathbf{V}_{k}^{(r, a) \mathrm{H}} \quad ; \text { with } \\
\boldsymbol{\Sigma}_{k}^{(r, a)} & =\operatorname{diag}\left(\sqrt{\lambda_{k, m}^{(r, a)}}\right) \quad m=1 \ldots \min \left(N_{\mathrm{R}}, N_{\mathrm{T}}\right) .
\end{aligned}
$$

The optimum, capacity-achieving, frequency-dependent precoding at the transmitter is given by the unitary matrix $\mathbf{V}_{k}^{(r, a)}$. If this precoding matrix $\mathbf{V}_{k}^{(r, a)}$ is used at the transmitter and also the optimum receive filter $\mathbf{U}_{k}^{(r, a) \mathrm{H}}$ is employed, the MIMO channel is separated into $\min \left(N_{\mathrm{R}}, N_{\mathrm{T}}\right)$ (with $N_{\mathrm{R}}$ denoting the number of receive antennas and $N_{\mathrm{T}}$ the number of transmit antennas) independent SISO channels each with a gain of $\sqrt{\lambda_{k, m}}, m=1 \ldots \min \left(N_{\mathrm{R}}, N_{\mathrm{T}}\right)$. The channel capacity is obtained by optimally distributing the available transmit power over these parallel SISO subchannels with unequal gain. The optimum power distribution $P_{k, m}^{(r, a)}$ is the solution of the following optimization problem:

$$
C^{(r, a)}=\max _{P_{k, m}^{(r, a)}} \frac{1}{K} \sum_{m=1}^{\min \left(N_{\mathrm{R}}, N_{\mathrm{T}}\right)} \sum_{k=1}^{K} \log _{2}\left(1+P_{k, m}^{(r, a)} \lambda_{k, m}^{(r, a)}\right)
$$

$$
\text { subject to } \sum_{m=1}^{\min \left(N_{\mathrm{R}}, N_{\mathrm{T}}\right)} \sum_{k=1}^{K} P_{k, m}^{(r, a)}=P_{\mathrm{t}} .
$$

Here, the second equation is a transmit power constraint that ensures an average transmit power proportional to the number of data subcarriers: $P_{\mathrm{t}}=K$. We calculate the power coefficients maximizing Equation (2) by using the water-filling algorithm described in [19, 20].

Equation (2) represents the average channel capacity per subcarrier in bit/s/Hz for a specific channel realization $r$ at a specific transmit power attenuation value $a$. In order to compare the capacity to the measured data throughput, Equation (2) has to be averaged over all $R$ measured channel realizations and scaled by the bandwidth $\frac{1}{T_{\mathrm{s}}}=5.76 \mathrm{MHz}$ of the WiMAX system:

$$
C^{(a)}=\frac{1}{T_{\mathrm{s}} \cdot R} \sum_{r=1}^{R} C^{(r, a)} .
$$

The resulting Equation (3) is a theoretical upper bound for the measured data throughput when the transmitter has perfect knowledge about the channel.

\section{B. Mutual Information}

The mutual information between a symbol vector transmitted over the $k$-th subcarrier of a MIMO OFDM system and the corresponding received symbol vector is given by [22, 23]:

$$
I_{k}^{(r, a)}=\log _{2}\left\{\operatorname{det}\left(\mathbf{I}+\frac{1}{\sigma_{\mathrm{n}}^{2}} \mathbf{H}_{k}^{(r, a)} \mathbf{H}_{k}^{(r, a) \mathrm{H}}\right)\right\} .
$$

The matrix $\mathbf{H}_{k}^{(r, a)}$ represents the MIMO channel coefficients, $\sigma_{\mathrm{n}}^{2}$ the noise variance, and $\mathbf{I}$ the identity matrix. If the transmitter has no knowledge about the channel, in theory a transmission rate corresponding to this mutual information can be achieved when Gaussian signals are transmitted.
For a specific transmit power attenuation value $a$, the average mutual information $I_{\text {avg }}^{(a)}$ per subcarrier is calculated by averaging over all sub carriers and the $R$ different channel realizations (receive antenna positions):

$$
I_{\text {avg }}^{(a)}=\frac{1}{K \cdot R} \sum_{r=1}^{R} \sum_{k=1}^{K} I_{k}^{(r, a)} .
$$

The average mutual information in Equation (5) is in a next step scaled by the bandwidth $\frac{1}{T_{\mathrm{s}}}$ of the WiMAX system. In this way, a theoretical upper bound for the measured data throughput is obtained when the transmitter does not perform precoding:

$$
I^{(a)}=\frac{1}{T_{\mathrm{s}}} I_{\mathrm{avg}}^{(a)} .
$$

\section{Achievable Throughput}

The rate given by the mutual information in Equation (6) can never be achieved by the WiMAX system since an OFDM signal requires also the transmission of a cyclic prefix to avoid intersymbol interference as well as the transmission of training symbols for synchronization and channel estimation. It is therefore convenient to introduce the achievable throughput $D_{\text {achievable }}^{(a)}$ that is based on mutual information but accounts for these inherent system losses. We define this achievable throughput for the transmit power attenuation value $a$ as

$$
D_{\text {achievable }}^{(a)}=\underbrace{\frac{1}{1+G} \cdot \frac{K}{K_{\mathrm{FFT}}} \cdot \frac{N_{\mathrm{data}}}{N_{\mathrm{OFDM}}}}_{1-L} \cdot I^{(a)},
$$

where $G$ (1/4 in our measurements) denotes the ratio between the cyclic prefix time and the useful OFDM symbol time, $K$ (192 in our measurements) the number of data subcarriers, $K_{\mathrm{FFT}}$ (256 in our measurements) the total number of subcarriers, $N_{\text {data }}$ (44 in our measurements) the number of OFDM data symbols, $N_{\text {OFDM }}$ (47 in our measurements) the total number of OFDM symbols in one transmission frame, and $I^{(a)}$ the mutual information as defined in Equation (6). Note that we introduced in Equation (7) also the system design loss $L$. In our measurements $D_{\text {achievable }}^{(a)} \approx 0.56 \cdot I^{(a)}$, thus $L=0.44$.

Equations (3), (6), and (7) will be used to calculate the performance bounds for the measured data throughput in Section IV.

\section{Measurement Setup}

For the measurements we utilized the Vienna MIMO Testbed described in [24-27]. The transmitter and receiver placing ${ }^{1}$ as well as the measurement procedure are explained in [28]. In case of MIMO transmission we utilize cross polarized antenna elements at the transmitter side and at the receiver side. The SISO performance was obtained as an average over the measured throughputs of all individual SISO subchannels of the MIMO system.

\footnotetext{
${ }^{1}$ Detailed transmitter and receiver positions for both scenarios can be downloaded for Google Earth at http://www.nt.tuwien.ac.at/fileadmin/data/ testbed/Vienna-and-Carinthia-TX-RX-GPS.kmz.
} 


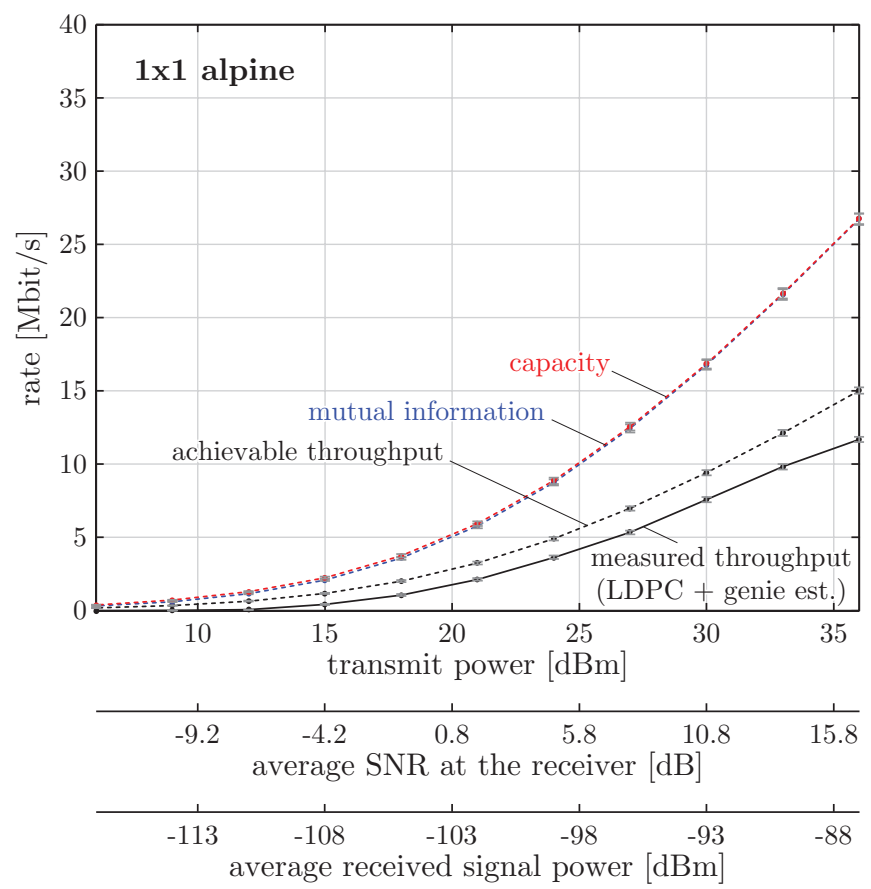

Fig. 1. $1 \times 1$ SISO system, alpine scenario: capacity, mutual information, achievable throughput, and measured throughput (genie-driven channel estimation and LDPC coding), measurement ID 2008-09-23.

\section{Measurement Results}

In all result figures, the dots represent the mean scenario throughputs, the vertical lines the corresponding 95\% confidence intervals for the mean [29], and the horizontal lines the corresponding $2.5 \%$ and $97.5 \%$ percentiles.

Figures 1 and 2 show the measurement results for the $1 \times 1$ SISO system and the $2 \times 2$ MIMO system in the alpine scenario. In each figure, four curves are plotted: the channel capacity defined in Equation (3), the mutual information defined in Equation (6), the achievable throughput defined in Equation (7), and the actually measured throughput of the WiMAX system. In case of the SISO system in Figure 1, the channel capacity is almost equal to the mutual information. This is due to the flat fading characteristic of the wireless channel with a very small mean RMS delay spread $^{2}$ of about $260 \mathrm{~ns}$ in the alpine scenario. In case of the MIMO system, the maximum improvement of the capacity over the mutual information is about $25 \%$. This gain is due to the spatial precoding and the optimum spatial water-filling. Due to the inherent losses of the OFDM system, the achievable throughput is $44 \%$ smaller than the mutual information in case of the SISO as well as the MIMO transmission. Comparing the achievable throughput to the measured throughput for LDPC coding and genie-driven channel estimation (the best performing combination) at $5 \mathrm{Mbit} / \mathrm{s}$, we measure an SNR loss of $2.4 \mathrm{~dB}$. This loss can be explained by Figure 3 showing the simulated throughput performance of the three (RS-CC, CTC, and LDPC) channel coding schemes over an Additive White Gaussian Noise (AWGN) channel. The seven steps of

\footnotetext{
${ }^{2}$ The mean RMS delay spread was calculated by averaging the RMS delay spreads of all channel realizations.
}

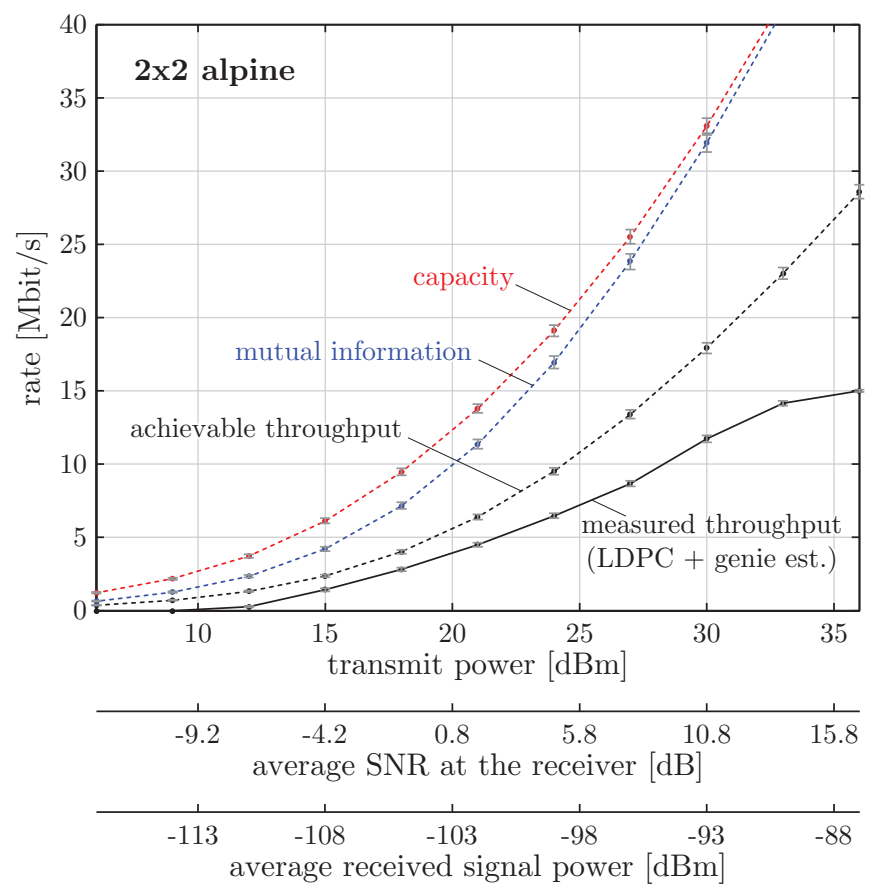

Fig. 2. $2 \times 2$ Alamouti-coded system, alpine scenario: capacity, mutual information, achievable throughput, and measured throughput (genie-driven channel estimation and LDPC coding), measurement ID 2008-09-23.

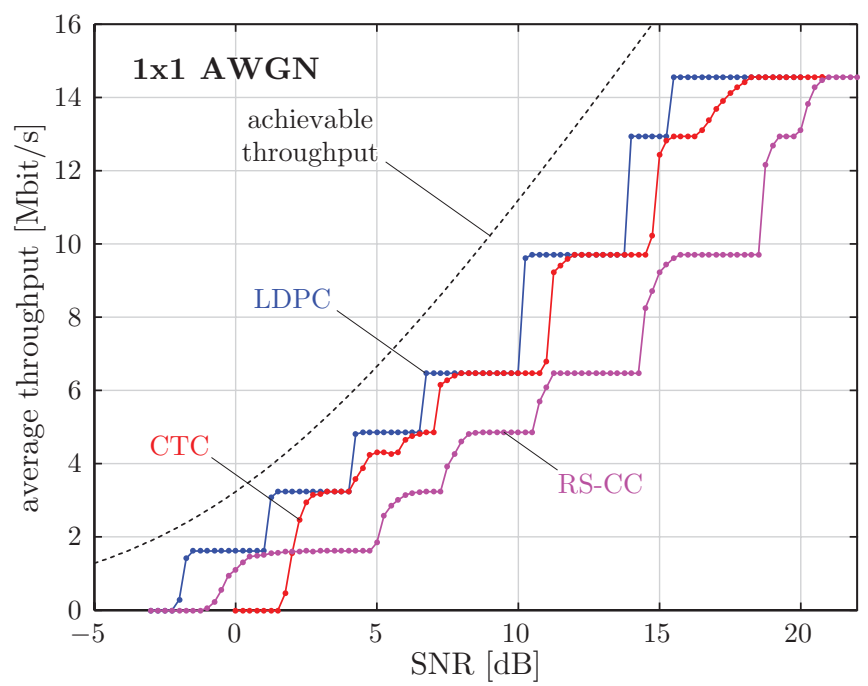

Fig. 3. Simulated throughput performance of the three channel codes in an AWGN channel.

the curves in Figure 3 correspond to the seven Adaptive Modulation and Coding (AMC) schemes defined in the WiMAX standard. In case of LDPC coding, the loss to the achievable throughput in the AWGN channel is between $1.8 \mathrm{~dB}$ and $3.8 \mathrm{~dB}$ at the third AMC scheme (at $4.85 \mathrm{Mbit} / \mathrm{s}$ ). In the measurement results shown in Figures 1 and 2, one throughput value is obtained by averaging over 484 channel realizations that have different instantaneous SNRs and thus different losses to the achievable throughput. On average, this leads to the measured loss of $2.4 \mathrm{~dB}$.

In Figure 2 we observe that the SNR loss of the measured throughput with respect to the achievable throughput increases 


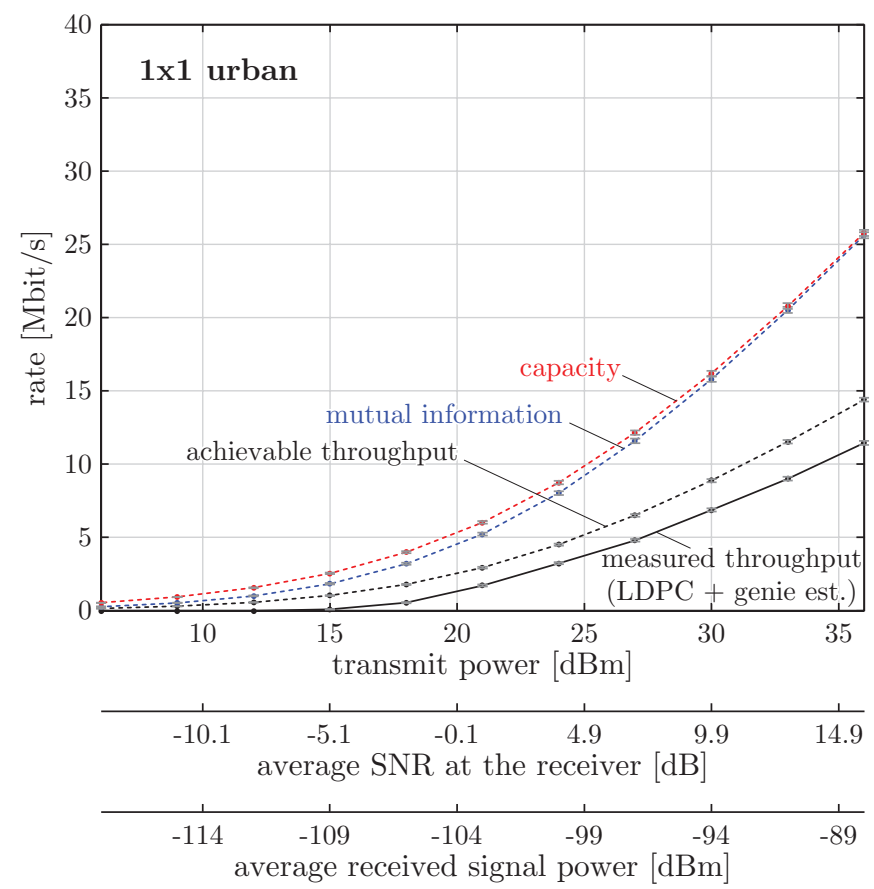

Fig. 4. $1 \times 1$ SISO system, urban scenario: capacity, mutual information, achievable throughput, and measured throughput (genie-driven channel estimation and LDPC coding), measurement ID 2009-01-15c.

with the SNR. This is because in the case of $2 \times 2$ transmission, the Alamouti code is not a full-rate full-diversity code and thus not optimal. A full-rate code, like for example the Golden code [30], could be used to obtain better performance [31].

Figures 4 and 5 show the measurement results for the $1 \times 1$ SISO and the $2 \times 2$ MIMO system in the urban scenario. In contrast to the alpine scenario, the capacity is slightly larger than the mutual information in case of the SISO transmission. This is due to the large mean RMS delay spread of about $1 \mu$ s resulting in a frequency selective channel. Therefore, the optimum power distribution over the full frequency band (we measured a WiMAX system with $5.76 \mathrm{MHz}$ sampling rate corresponding to a symbol duration of $174 \mathrm{~ns}$ ) is not flat anymore and water-filling can increase the possible transmission rate. All other results of the urban scenario are qualitatively and quantitatively the same as in the alpine scenario.

For the alpine scenario, Figure 6 shows the SNR losses of different channel estimators and channel codes with respect to the best performing combination: genie-driven channel estimation with LDPC coding. The performance of the $2 \times 2$ Alamouti coded system depends much more on the type of channel estimator implemented than the SISO system does. This can be explained by the fact that in case of Alamouti transmission, the available transmit power and thus also the training signal power is equally distributed over the transmit antennas. Therefore, only half the training signal power is available per channel coefficient to be estimated. As a consequence, the channel estimation performance as well as the throughput performance is poorer than in the SISO transmission.

Figure 7 shows the SNR losses for the urban scenario. The losses of the LDPC and CTC channel coding schemes are similar to the losses determined in the alpine scenario in Figure 6.

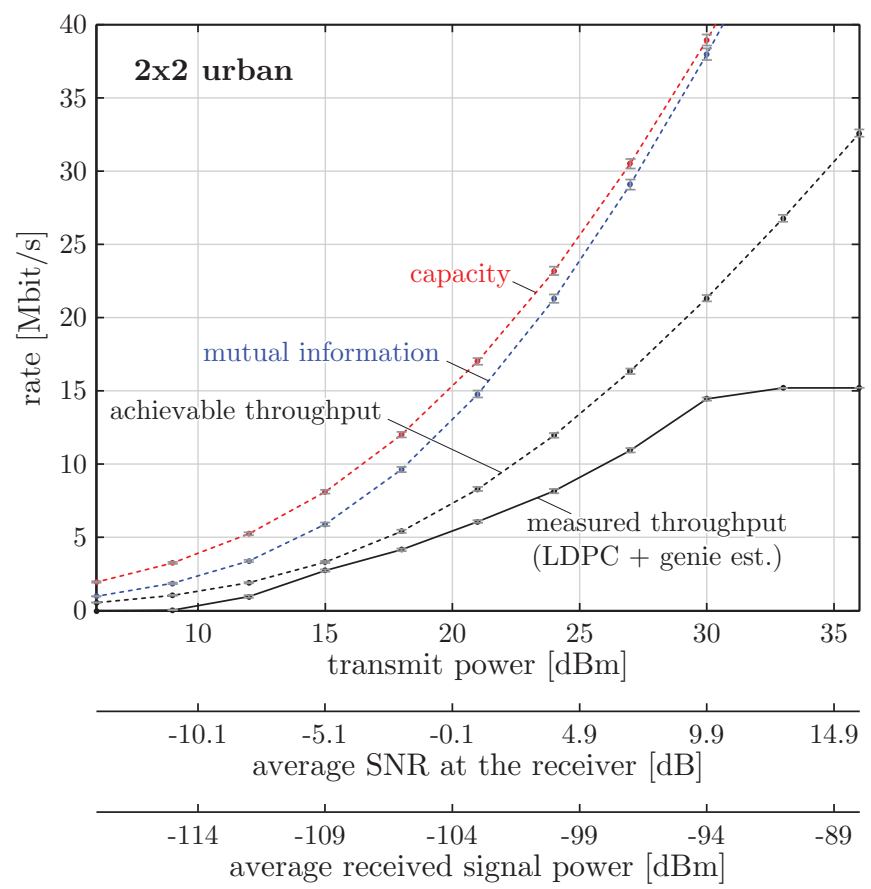

Fig. 5. $2 \times 2$ Alamouti-coded system, urban scenario: capacity, mutual information, achievable throughput, and measured throughput (genie-driven channel estimation and LDPC coding), measurement ID 2009-01-15c.

For the RS-CC channel coding scheme, however, a significant difference is observed. Especially when a SISO transmission is performed, the SNR loss with RS-CC is significantly larger than in the alpine scenario. The reason for this increased loss lies in the frequency selective nature of the wireless channel in the urban scenario. The large delay spread causes deep fades which in turn cause a poor performance of the RSCC channel code. In case of $2 \times 2$ Alamouti transmission, the spatial diversity reduces the depth of the fades in the frequency response, resulting in an SNR loss of the RS-CC in the urban scenario similar to the SNR loss in the alpine scenario. These observations were confirmed by simulations in [16, Appendix A.2., Figures 14 and 15].

In summary, depending on the actual algorithms implemented, the losses to the best performing scheme can be very large. If only a very simple system with RS-CC coding and LS channel estimation is implemented, the loss with respect to the best performing combination was measured to be as large as $6 \mathrm{~dB}$ in SNR.

\section{Conclusions}

In this paper, throughput measurement results of the IEEE 802.16-2004 physical layer are presented. The measured throughput is compared to the channel capacity, the mutual information, and the achievable throughput that accounts for the inherent system losses. We find that for the SISO system with genie-driven channel estimation, the loss of the measured throughput to the achievable throughput is only given by the loss of the channel coding scheme and the limited number of AMC schemes. Depending on the channel code and the channel estimator employed, the SNR losses at a throughput of $5 \mathrm{Mbit} / \mathrm{s}$ grow up to $6 \mathrm{~dB}$. 


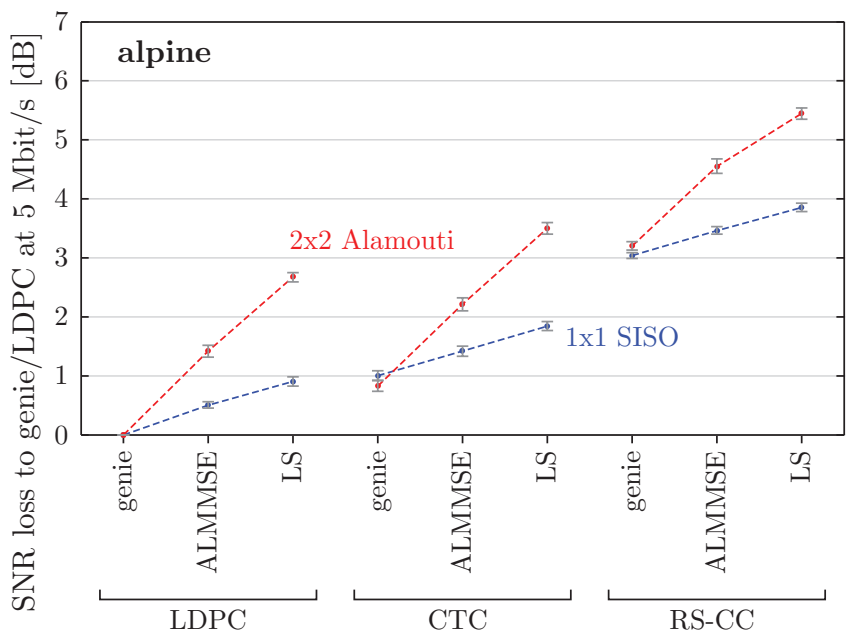

Fig. 6. $1 \times 1$ SISO and $2 \times 2$ Alamouti-coded system in the alpine scenario SNR losses of the different channel estimators and channel codes with respect to the genie-driven channel estimator and the LDPC code, measurement ID 2008-09-23.

\section{ACKNOWLEDGMENTS}

This work has been funded by the Christian Doppler Laboratory for Wireless Technologies for Sustainable Mobility, the Institute of Communications and Radio-Frequency Engineering, KATHREIN-Werke KG, and mobilkom austria AG. It has also been supported by the Xunta de Galicia, the Ministerio de Ciencia e Innovación of Spain, and the FEDER funds of the European Union under the grants PGIDIT06TIC10501PR, TEC2007-68020-C04-01 and CSD2008-00010.

\section{REFERENCES}

[1] C. Hoymann, "Analysis and performance evaluation of the OFDM-based metropolitan area network IEEE 802.16," Computer Networks, Selected Paper from the European Wireless 2004 Conference, vol. 49, no. 3, pp. 341-363, 2005.

[2] M. A. Hasan, "Performance evaluation of WiMAX/IEEE 802.16 OFDM physica layer," Master's thesis, Helsinki University of Technology, Department of Electrical and Communications Engineering, Communications Laboratory, Jun. 2007. [Online]. Available: http://lib.tkk.fi/Dipl/2007/urn009599.pdf

[3] I. Koffman and V. Roman, "Broadband wireless access solutions based on OFDM access in IEEE 802.16," IEEE Communications Magazine, vol. 40, no. 4, pp. 96-103, Apr. 2002.

[4] C. Eklund, R. Marks, K. Stanwood, and S. Wang, "IEEE standard 802.16: A technical overview of the WirelessMAN air interface for broadband wireless access," IEEE Communications Magazine, vol. 40, no. 6, pp. 98-107, Jun. 2002.

[5] D.-H. Cho, J.-H. Song, M.-S. Kim, and K.-J. Han, "Performance analysis of the IEEE 802.16 wireless metropolitan area network," in Proc. 1st International Conference on Distributed Frameworks for Multimedia Applications 2005 (DFMA 2005), Feb. 2005, pp. 130-136

[6] F. Wang, A. Ghosh, R. Love, K. Stewart, R. Ratasukt, R. Bachu, Y. Sun, and Q. Zhao, "IEEE 802.16e system performance: analysis and simulations," in Proc. 16th IEEE International Symposium on Personal, Indoor and Mobile Radio Communications (PIMRC 2005), Berlin, Germany, Sep. 2005.

[7] B. Muquet, E. Biglieri, and H. Sari, "MIMO link adaptation in mobile WiMAX systems," in Proc. IEEE Wireless Communications and Networking Conference 2007 (WCNC 2007), Hong Kong, Mar. 2007.

[8] T. H. Chan, C. Y. Cheung, M. Hamdi, and M. Ma, "Overview of rate adaptation algorithms based on MIMO technology in WiMAX networks" in Proc. IEEE Mobile WiMAX Symposium 2007, Orlando, FL, USA, Mar. 2007.

[9] M. Malkowski, "Link-level comparison of IP-OFDMA (mobile WiMAX) and UMTS HSDPA," in Proc. IEEE 18th International Symposium on Personal, Indoor and Mobile Radio Communications 2007 (PIMRC 2007), 2007.

[10] O. Grøndalen, P. Grønsund, T. Breivik, and P. Engelstad, "Fixed WiMAX field trial measurements and analyses," in Proc. 16th IST Mobile and Wireless Communications Summit, 2007, Jul. 2007.

[11] P. Grønsund, P. Engelstad, T. Johnsen, and T. Skeie, "The physical performance and path loss in a fixed WiMAX deployment," in Proc. International Conference on Wireless Communications and Mobile Computing 2007 (IWCMC 2007), 2007, pp. 439-444.

[12] N. Scalabrino, F. De Pellegrini, R. Riggio, A. Maestrini, C. Costa, and I. Chlamtac, "Measuring the quality of VoIP traffic on a WiMAX testbed," in Proc. 3rd International Conference on Testbeds and Research Infrastructure for the Development of Networks and Communities (TridentCom 2007), May 2007.

[13] K. Pentikousis, J. Pinola, E. Piri, and F. Fitzek, "An experimental investigation of VoIP and video streaming over fixed WiMAX," in Proc. 6th International Symposium on Modeling and Optimization in Mobile, Ad Hoc, and Wireles Networks and Workshops 2008 (WiOPT 2008), Apr. 2008, pp. 8-15.



Fig. 7. $1 \times 1$ SISO and $2 \times 2$ Alamouti-coded system in the urban scenario: SNR losses of the different channel estimators and channel codes with respect to the genie-driven channel estimator and the LDPC code, measurement ID 2009-01-15c.

[14] A. Durantini, M. Petracca, and F. Ananasso, "Experimental evaluation of IEEE 802.16 WiMAX performances at $2.5 \mathrm{GHz}$ band," in Proc. International Wireles Communications and Mobile Computing Conference 2008 (IWCMC 2008), Aug. 2008, pp. 338-343.

[15] H. Sampath, S. Talwar, J. Tellado, V. Erceg, and A. Paulraj, "A fourth-generation MIMO-OFDM broadband wireless system: design, performance, and field trial results," IEEE Communications Magazine, vol. 40, no. 9, pp. 143-149, Sep. 2002

[16] C. Mehlführer, S. Caban, and M. Rupp, "Experimental evaluation of adaptive modulation and coding in MIMO WiMAX with limited feedback," EURASIP Journal on Advances in Signal Processing, Special Issue on MIMO Systems with Limited Feedback, vol. 2008, Article ID 837102, 2008.

[17] IEEE, "IEEE standard for local and metropolitan area networks; part 16: Air interface for fixed broadband wireless access systems, IEEE Std. 802.16-2004," Oct. 2004. [Online]. Available: http://standards.ieee.org/getieee802/download/802. 16-2004.pdf

[18] C. Mehlführer, S. Caban, and M. Rupp, "An accurate and low complex channel estimator for OFDM WiMAX," in Proc. 3rd International Symposium on Communications, Control and Signal Processing (ISCCSP 2008), St. Julians, Malta, Mar. 2008, pp. 922-926.

[19] G. G. Raleigh and J. M. Cioffi, "Spatio-temporal coding for wireless communication," IEEE Transactions on Communications, vol. 46, no. 3, pp 357-366, Mar. 1998

[20] A. Paulraj, R. Nabar, and D. Gore, Introduction to Space-Time Wireless Communications, 1 st ed. Cambridge University Press, 2003.

[21] T. K. Moon and W. C. Stirling, Mathematical Methods and Algorithms for Signal processing, 1st ed. Upper Saddle River, NJ: Prentice Hall, 2000.

[22] G. J. Foschini and M. J. Gans, "On limits of wireless communication in a fading environment when using multiple antennas," Wireless Personal Communications, vol. 6 , no. 3, pp. 311-335, 1998

[23] I. E. Telatar, "Capacity of multi-antenna gaussian channels," European Transaction on Telecommunications, 1999, Technical Memorandum, Bell Laboratories, Lucen Technologies, vol. 10, no. 6, pp. 585-595, Oct. 1998.

[24] S. Caban, "Testbed-based evaluation of mobile communication systems," Ph.D dissertation, Technische Universität Wien, Institut für Nachrichtentechnik und Hochfrequenztechnik, Sep. 2009, supervisor: Markus Rupp.

[25] S. Caban, C. Mehlführer, R. Langwieser, A. L. Scholtz, and M. Rupp, "Vienn MIMO testbed," EURASIP Journal on Applied Signal Processing, Special Issue on Implementation Aspects and Testbeds for MIMO Systems, vol. 2006, Article ID 54868, 2006.

[26] C. Mehlführer, S. Geirhofer, S. Caban, and M. Rupp, "A flexible MIMO testbed with remote access," in Proc. 13th European Signal Processing Conference (EUSIPCO 2005), Antalya, Turkey, Sep. 2005.

[27] M. Rupp, S. Caban, and C. Mehlführer, "Challenges in building MIMO testbeds," in Proc. 15th European Signal Processing Conference (EUSIPCO 2007), Poznań, Poland, Sep. 2007.

[28] S. Caban, C. Mehlführer, G. Lechner, and M. Rupp, "Testbedding MIMO HSDPA and WiMAX," in Proc. 70th IEEE Vehicular Technology Conference (VTC2009-Fall), Anchorage, AK, USA, Sep. 2009

[29] B. Efron and D. V. Hinkley, An Introduction to the Bootstrap (CRC Monograph on Statistics \& Applied Probability 57), 1st ed. Chapman \& Hall, 1994.

[30] J.-C. Belfiore, G. Rekaya, and E. Viterbo, "The golden code: a $2 \times 2$ full-rate spacetime code with nonvanishing determinants," IEEE Transactions on Information Theory, vol. 51, no. 4, pp. 1432-1436, Apr. 2005.

[31] A. Hottinen, Y. Hong, E. Viterbo, C. Mehlführer, and C. Mecklenbräuker, "A comparison of high rate algebraic and non-orthogonal STBCs," in Proc. ITG/IEEE Workshop on Smart Antennas (WSA 2007), Vienna, Austria, Feb. 2007. 
Copyright 2001 SS\&C. Published in the Conference Record of the Fourtythird Asilomar Conference on Signals, Systems and Computers, 2009, November 1-4, 2009, Pacific Grove, CA, USA.

Reference:

C. Mehlführer, S. Caban, J. A. García-Naya, and M. Rupp, "Throughput and capacity of MIMO WiMAX," in Conference Record of the 43rd Asilomar Conference on Signals, Systems and Computers, Pacific Grove, CA, USA, Nov. 2009.

[Online]. Available: http://publik.tuwien.ac.at/files/PubDat_178050.pdf

BibTeX:

e InProceedings $\{$ Mehlfuehrer_Asilomar2009,

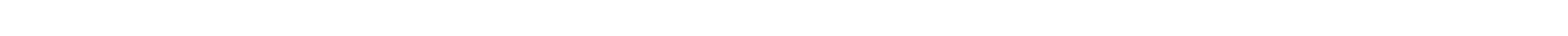

title $=$

\{Throughput and Capacity of \{MIMO\} \{WiMAX\}\},

booktitle $=\quad$ Conference Record of the 43rd Asilomar Conference on Signals, Systems and Computers\},

month $=$

year $=$ nov,

address $=\quad\{$ Pacific Grove, CA, USA $\}$,

url $=$

\}

\{http://publik.tuwien.ac.at/files/PubDat_178050.pdf\}, 